\title{
Data-driven cross-talk modeling of beam losses in LHC collimators
}

\author{
Gabriella Azzopardi, ${ }^{1,2, *}$ Belen Salvachua, ${ }^{1}$ and Gianluca Valentino ${ }^{2}$ \\ ${ }^{1}$ CERN, Geneva, Switzerland \\ ${ }^{2}$ University of Malta, Msida, Malta
}

(Received 12 June 2019; published 8 August 2019)

\begin{abstract}
The Large Hadron Collider at CERN is equipped with a collimation system to intercept potentially dangerous beam halo particles before they damage its sensitive equipment. The collimator settings are determined following a beam-based alignment procedure, in which the collimator jaws are moved towards the beam until losses appear in the beam loss monitors. When the collimator reaches the beam envelope, beam losses propagate mainly in the direction of the beam and are, therefore, also observed by other nearby beam loss monitors. This phenomenon is known as cross talk. Due to this, collimators are aligned sequentially to be able to identify which losses are generated by which collimator, such that any cross talk across beam loss monitors positioned close to each other is avoided. This paper seeks to quantify the levels of cross-talk observed by beam loss monitors when multiple collimators are moving, to be able to determine the actual beam loss signals generated by their corresponding collimators. The results obtained successfully predicted the amount of cross-talk observed for each of the cases tested in this study. This was then extended to predict loss map case studies and the proton impacts at each collimator by comparing them to simulations.
\end{abstract}

DOI: 10.1103/PhysRevAccelBeams.22.083002

\section{INTRODUCTION}

The Large Hadron Collider (LHC) at CERN is designed to collide two counterrotating beams with an unprecedented center-of-mass energy of $14 \mathrm{TeV}$ [1]. It is made up of eight arcs containing superconducting magnets and eight straight sections which are referred to as insertion regions (IRs). Four IRs house the four main experiments, ATLAS, ALICE, CMS, and LHCb, which are located at the points where the beams are brought into collisions, referred to as interaction points (IPs) [2].

The LHC makes use of a collimation system to scatter and absorb beam losses from normal and abnormal conditions, to protect the superconducting magnets and other sensitive equipment from any damage [3]. The collimation system consists of 100 collimators able to provide a 99.998\% cleaning efficiency of all halo particles.

Collimators are set up in the form of a hierarchy, whereby the primary collimators (TCPs) are placed closest to the beam to intercept the primary halo particles; secondary collimators (TCSGs) are further retracted to clean secondary particles; the absorbers (TCLAs) absorb the remaining showers; and the tertiary collimators (TCTs)

\footnotetext{
*gabriella.azzopardi@cern.ch
}

Published by the American Physical Society under the terms of the Creative Commons Attribution 4.0 International license. Further distribution of this work must maintain attribution to the author(s) and the published article's title, journal citation, and DOI. are installed close to the LHC's experiments to provide local protection of the focusing triplet superconducting magnet. The collimators are mainly concentrated in two dedicated cleaning IRs, IR3 for momentum cleaning and IR7 for betatron cleaning, as can be seen in Fig. 1.

A collimator is made up of two parallel blocks, referred to as jaws, inside a vacuum tank. The jaw corners can be moved individually using dedicated stepping motors and are positioned around the beam with one jaw on each side. Collimators are oriented such that they are able to clean in either the horizontal $(\mathrm{H})$, vertical $(\mathrm{V})$, or skew $(\mathrm{S})$ plane. Each collimator has a dedicated ionization chamber, part of the beam loss monitoring (BLM) system [4], positioned immediately downstream to detect beam losses generated when halo particles impact the collimator jaws, as shown in Fig. 2. These losses are proportional to the amount of beam intercepted by the collimator jaws, in units of Gy/s.

The LHC collimation system needs to be aligned around the beam to ensure the correct hierarchy. This hierarchy is regularly checked by observing the spatial distribution of the measured losses around the ring (known as a loss map), in which the losses are generated following an intentional excitation of the beam in the transverse plane.

The collimation alignment procedure involves individually moving the collimator jaws towards the beam, until a spike is observed in the BLM reading. When a particle reaches the primary collimator, it can either have an inelastic interaction and get absorbed, thus starting a hadronic and electromagnetic shower that can be detected by several BLMs (including BLMs in the other beam), 


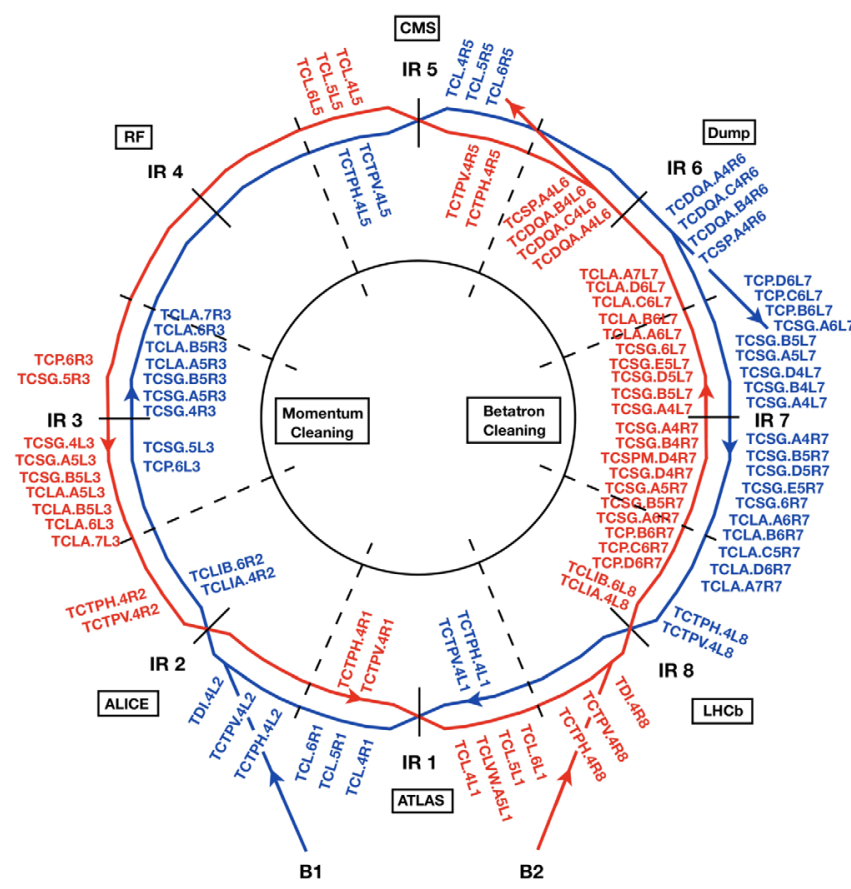

FIG. 1. The present collimation system layout with the majority of the collimators in IR3 and IR7. Other collimators are placed in the dump and transfer regions and in the experimental IPs to protect the triplet magnets. The naming convention used is the official LHC collimator layout name.

or interact elastically, thus changing its momentum, and continue its trajectory. In both cases, additional signals, referred to as cross talk, could be measured by BLM devices placed downstream from the initial interactions.

It would be beneficial to quantify the cross-talk experienced at a selected beam loss monitor, in cases such as collimator alignment or predicting the main plane of losses. This motivated a study on the cross talk between collimators in IR7, and this paper seeks to quantify the level of cross talk in BLM signals, following dedicated beam tests performed in the LHC.

The outline of the paper is as follows. The procedure and measurements obtained from the LHC are described in Sec. II. Sections III and IV explain the calculations

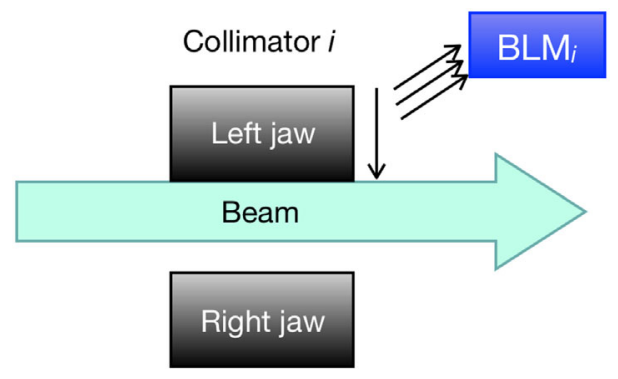

FIG. 2. The jaws of collimator $i$ around the beam, with its left jaw scraping the beam halo, and the showers are detected by the corresponding BLM detector downstream. and analysis involved in quantifying the level of cross-talk observed during the beam tests. Section V seeks to validate the cross-talk factors obtained, by comparing the results to the measured signals. Section VI applies the same analysis to loss map case studies. Finally, Sec. VII seeks to calculate the number of protons per second that impacted the collimators, and Sec. VIII compares these results to particletracking simulations.

\section{MEASUREMENTS FROM BEAM TESTS}

The measurements for this study were taken at injection, with a beam energy of $450 \mathrm{GeV}$, focusing on collimators in IR7 in the two beams [5]. Each beam was injected with 25 pilot bunches, each having an average bunch intensity of $6 \times 10^{9}$ protons per bunch.

The measurement procedure started by positioning only one collimator close to the beam, while the rest of the hierarchy was retracted (individual cases). At this point, a selected bunch was excited using the transverse damper [6], until it was fully scraped away at the selected collimator. This was repeated for each collimator listed in Table I, which identifies each collimator by a label and lists the corresponding cleaning plane (vertical or horizontal). Each collimator was closed by moving it to its injection setting (refer to Table II), and, for cases when these settings were too far from the beam (e.g., TCLAs), the collimators were manually moved closer to the beam.

TABLE I. Individual cases of collimators that were set as the primary bottleneck and the cleaning plane.

\begin{tabular}{lccc}
\hline \hline ID & Collimator & Cleaning Plane & Case \\
\hline B1V-1 & TCP.D6L7.B1 & Vertical & 1 \\
B1V-2 & TCSG.D4L7.B1 & Vertical & 2 \\
B1V-3 & TCLA.A6R7.B1 & Vertical & 3 \\
B1V-4 & TCLA.C6R7.B1 & Vertical & 4 \\
B1H-1 & TCP.C6L7.B1 & Horizontal & 1 \\
B1H-2 & TCSG.B4L7.B1 & Horizontal & 2 \\
B1H-3 & TCSG.6R7.B1 & Horizontal & 3 \\
B1H-4 & TCLA.B6R7.B1 & Horizontal & 4 \\
B1H-5 & TCLA.D6R7.B1 & Horizontal & 5 \\
B1H-6 & TCLA.A7R7.B1 & Horizontal & 6 \\
B2V-1 & TCP.D6R7.B2 & Vertical & 1 \\
B2V-2 & TCSG.D4R7.B2 & Vertical & 2 \\
B2V-3 & TCSPM.D4R7.B2 & Vertical & 3 \\
B2V-4 & TCLA.A6L7.B2 & Vertical & 4 \\
B2V-5 & TCLA.C6L7.B2 & Vertical & 5 \\
B2H-1 & TCP.C6R7.B2 & Horizontal & 1 \\
B2H-2 & TCSG.B4R7.B2 & Horizontal & 2 \\
B2H-3 & TCSG.6L7.B2 & Horizontal & 3 \\
B2H-4 & TCLA.B6L7.B2 & Horizontal & 4 \\
B2H-5 & TCLA.D6L7.B2 & Horizontal & 5 \\
B2H-6 & TCLA.A7L7.B2 & Horizontal & 6 \\
\hline \hline
\end{tabular}


TABLE II. List of 2018 injection settings for collimation.

\begin{tabular}{lccc}
\hline \hline Collimator & IR & Orientation & Setting $(\sigma)$ \\
\hline Primary (TCP) & 7 & H/V/S & 5.7 \\
Secondary (TCSG) & 7 & H/V/S & 6.7 \\
Absorber (TCLA) & 7 & $\mathrm{H} / \mathrm{V}$ & 10.0 \\
Primary (TCP) & 3 & $\mathrm{H}$ & 8.0 \\
Secondary (TCSG) & 3 & $\mathrm{H} / \mathrm{V}$ & 9.3 \\
Absorber (TCLA) & 3 & $\mathrm{H}$ & 12.0 \\
Secondary (TCSP) & 6 & $\mathrm{H}$ & 7.5 \\
Dump prot. (TCDQ) & 6 & $\mathrm{H}$ & 8.0 \\
Tertiary (TCT) & $1 / 2 / 5 / 8$ & $\mathrm{H} / \mathrm{V}$ & 13.0 \\
\hline \hline
\end{tabular}

TABLE III. Combined cases of collimators that were set as a primary bottleneck with a hierarchy and the cleaning plane.

\begin{tabular}{lcc}
\hline \hline ID & Plane & Case \\
\hline $\mathrm{B} 1 \mathrm{~V}-1+2$ & Vertical & $1+2$ \\
$\mathrm{~B} 1 \mathrm{~V}-1+2+3$ & Vertical & $1+2+3$ \\
$\mathrm{~B} 1 \mathrm{~V}-1+2+3+4$ & Vertical & $1+2+3+4$ \\
$\mathrm{~B} 1 \mathrm{H}-1+2$ & Horizontal & $1+2$ \\
$\mathrm{~B} 1 \mathrm{H}-1+2+3$ & Horizontal & $1+2+3$ \\
$\mathrm{~B} 1 \mathrm{H}-1+2+3+4$ & Horizontal & $1+2+3+4$ \\
$\mathrm{~B} 1 \mathrm{H}-1+2+3+4+5$ & Horizontal & $1+2+3+4+5$ \\
$\mathrm{~B} 1 \mathrm{H}-1+2+3+4+5+6$ & Horizontal & $1+2+3+4+5+6$ \\
$\mathrm{~B} 2 \mathrm{~V}-1+2$ & Vertical & $1+2$ \\
$\mathrm{~B} 2 \mathrm{~V}-1+2+3$ & Vertical & $1+2+3$ \\
$\mathrm{~B} 2 \mathrm{~V}-1+2+3+4$ & Vertical & $1+2+3+4$ \\
$\mathrm{~B} 2 \mathrm{~V}-1+2+3+4+5$ & Vertical & $1+2+3+4+5$ \\
$\mathrm{~B} 2 \mathrm{H}-1+2$ & Horizontal & $1+2$ \\
$\mathrm{~B} 2 \mathrm{H}-1+2+3$ & Horizontal & $1+2+3$ \\
$\mathrm{~B} 2 \mathrm{H}-1+2+3+4$ & Horizontal & $1+2+3+4$ \\
$\mathrm{~B} 2 \mathrm{H}-1+2+3+4+5$ & Horizontal & $1+2+3+4+5$ \\
B2H-1 $+2+3+4+5+6$ & Horizontal & $1+2+3+4+5+6$ \\
\hline \hline
\end{tabular}

This was followed by exciting the beam with various combinations of closed collimators positioned close to the beam (combined cases). For each combined case, the closed collimators followed the injection hierarchy. Table III lists the combined cases used, by combining the IDs from Table I; e.g., B1V-1 +2 indicates the combined case of collimators B1V-1 and B1 V-2 positioned close to the beam, as shown in Fig. 3.

\section{MATHEMATICAL FORMULATION}

The signal recorded by the BLM at collimator $j\left(S_{j}\right)$ is composed of the beam losses generated by collimator $j$ and upstream collimators (the latter resulting in cross talk). Therefore, it is necessary to quantify the level of cross talk present in $S_{j}$. This can be done using the BLM signal contribution of each individual upstream collimator $i$, without the contribution from other collimators, i.e., without cross talk $\left(S_{i}^{\prime}\right)$.

For $n$ collimators $(1,2, \ldots, n)$, the signal recorded by the BLM at the most downstream collimator $n$ can be defined using a general recursive equation:

$S_{n}=\sum_{i=1}^{n} a_{n i} S_{i}^{\prime}, \quad a_{n n}=1, \quad$ if $i>n: a_{n i}=0$,

where $a_{n i}$ is the cross-talk factor quantifying the effect of a closed upstream collimator $i$ on collimator $n$. For a set of $n$ collimators, this system of equations can be represented by the triangular matrix:

$$
\left[\begin{array}{c}
S_{1} \\
S_{2} \\
S_{3} \\
\vdots \\
S_{n}
\end{array}\right]=\left[\begin{array}{ccccc}
1 & 0 & 0 & \cdots & 0 \\
a_{21} & 1 & 0 & \cdots & 0 \\
a_{31} & a_{32} & 1 & \cdots & 0 \\
\vdots & \vdots & \vdots & \ddots & \vdots \\
a_{n 1} & a_{n 2} & a_{n 3} & \cdots & 1
\end{array}\right]\left[\begin{array}{c}
S_{1}^{\prime} \\
S_{2}^{\prime} \\
S_{3}^{\prime} \\
\vdots \\
S_{n}^{\prime}
\end{array}\right] .
$$

To calculate the cross-talk factors, the individual cases with each collimator closed individually must first be analyzed. As an example, two individual cases (case 1 and case 2 shown in Fig. 3) are used to calculate the crosstalk factors on the next open downstream collimator (3), to be able to predict the signal measured by its BLM:

$$
S_{3}=\mathbf{a}_{31} \cdot S_{1}^{\prime}+\mathbf{a}_{32} \cdot S_{2}^{\prime}+a_{33} \cdot S_{3}^{\prime} .
$$

For each individual case, the contributions from other collimators are zero as they are completely retracted. For

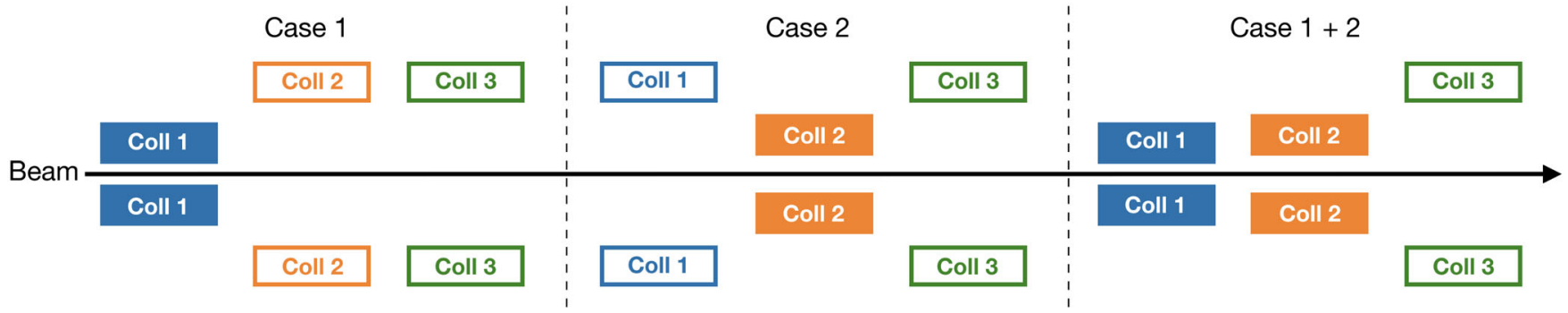

FIG. 3. Simplified diagram of three collimators (coll 1, coll 2, and coll 3), each one further downstream than the previous. Case 1 and case 2 individually close coll 1 and coll 2 , respectively, while case $1+2$ closes both collimators simultaneously. 
case 1 , the signal without cross talk is $0\left(S_{j}^{\prime}=0\right)$ for $j \neq 1$; therefore, all the coefficients $a_{j 1}$ can be calculated as follows.

Case 1: $S_{2}^{\prime}=S_{3}^{\prime}=0$

$$
\begin{array}{ll}
S_{1}=a_{11} \cdot S_{1}^{\prime}, & a_{11}=\frac{S_{1}}{S_{1}}=1, \\
S_{2}=a_{21} \cdot S_{1}^{\prime}, & a_{21}=\frac{S_{2}}{S_{1}}, \\
S_{3}=a_{31} \cdot S_{1}^{\prime}, & \mathbf{a}_{31}=\frac{S_{3}}{S_{1}} .
\end{array}
$$

Similarly, for case 2 , the signal without cross talk is 0 $\left(S_{j}^{\prime}=0\right)$ for $j \neq 2$ :

Case 2: $S_{1}^{\prime}=S_{3}^{\prime}=0$

$$
\begin{array}{ll}
S_{1}=a_{11} \cdot S_{1}^{\prime}=0, & \\
S_{2}=a_{22} \cdot S_{2}^{\prime}, & a_{22}=\frac{S_{2}}{S_{2}}=1, \\
S_{3}=a_{32} \cdot S_{2}^{\prime}, & \mathbf{a}_{32}=\frac{S_{3}}{S_{2}} .
\end{array}
$$

As a result, the cross-talk factors for Eq. (3) are calculated using Eqs. (4c) and (5c). This procedure can be applied recursively such that cases 1,2 , and 3 can be used to predict the signal observed by a fourth collimator and so on.

\section{BLM COEFFICIENT CALCULATION}

Beam tests for all of the collimator configurations were performed to determine the cross-talk factors and, therefore, predict the losses recorded by the first downstream collimator BLM after the last closed collimator. As an example, Fig. 4 presents the BLM signals recorded using three collimator configurations in the vertical plane of beam 1 (B1V-1, B1V-2, and B1V-1 + 2). The cross-talk factors determined for TCP.D6L7.B1 and TCSG.D4L7.B1 are then used to predict the cross-talk observed at TCLA.A6R7.B1.

From this figure, one can qualitatively observe the following. (i) Case B1V-1 shows that most of the particles are lost at TCP.D6L7.B1 when it acts as the primary bottleneck. The other two monitored collimators were retracted; therefore, the losses recorded by their BLMs derive from the protons lost at the primary collimator. (ii) Case $\mathrm{B} 1 \mathrm{~V}-2$ shows the losses generated when TCSG.D4L7.B1 is moved in; therefore, no losses are observed at TCP.D6L7.B1 (that is now retracted), but higher losses are recorded at TCLA.A6R7.B1. (iii) Case $\mathrm{B} 1 \mathrm{~V}-1+2$ is a combined case where beam losses occur at TCP.D6L7.B1 and TCSG.D4L7.B1 with different ratios. The aim is to predict the signal and compare it with the measured at TCLA.A6R7.B1, for this configuration.

Figure 5 shows the loss maps generated using the same three configurations, focusing solely on IR7 collimators in both beam 1 and beam 2, to show that cross talk can affect both beams. Finally, Fig. 6 highlights the linear correlation between the collimators' $100 \mathrm{~Hz}$ BLM signals, such that the linear fits can be used to calculate the cross-talk factors. On the other hand, this linearity between the BLM signals verifies that the equations in Sec. III can be used. Therefore, the BLM signals from all configurations are used in these equations to calculate the cross-talk factors, whereby each signal is integrated to eliminate any fluctuations between points. In both cases, the factors obtained are equivalent within a reasonable margin.

The resulting cross-talk factors for the vertical plane in beam 1 are displayed in Fig. 7, and a complete table

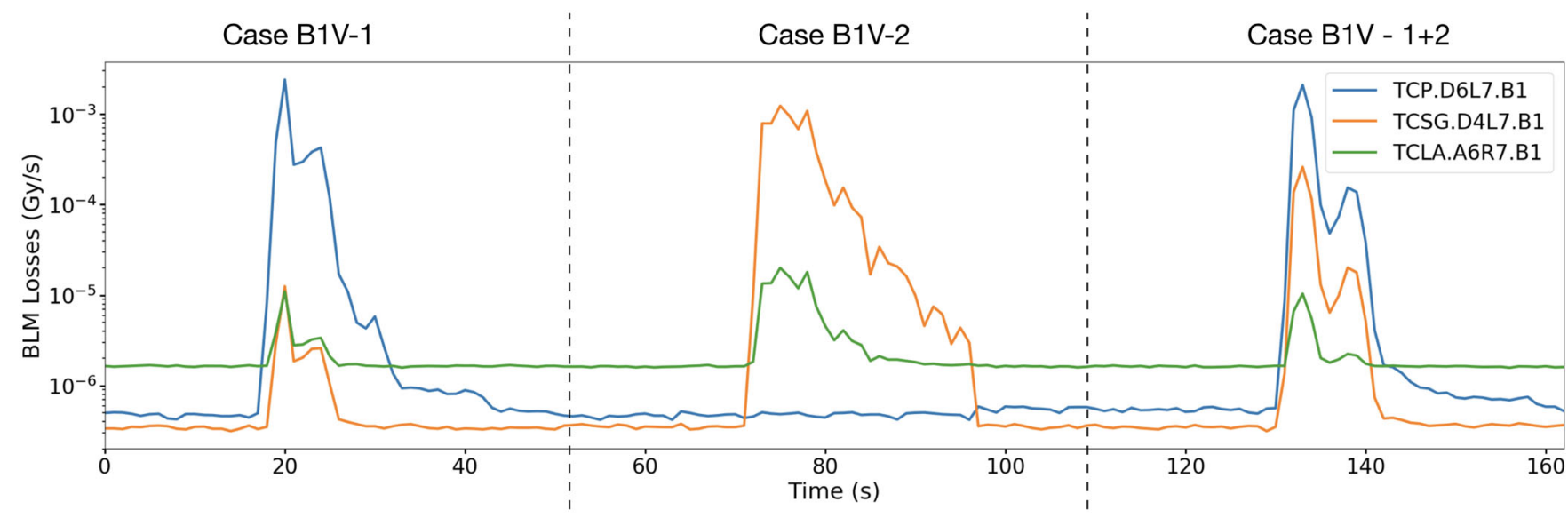

FIG. 4. Series of beam loss signals recorded for a subset of collimators in the vertical plane in beam 1. Case B1V-1 and case B1V-2 involve individually closing TCP.D6L7.B1 and TCSG.D4L7.B1, respectively, while case B1V-1 + 2 involves closing both collimators simultaneously, to ultimately predict the cross-talk experienced by the most downstream collimator, TCLA.A6R7.B1. 


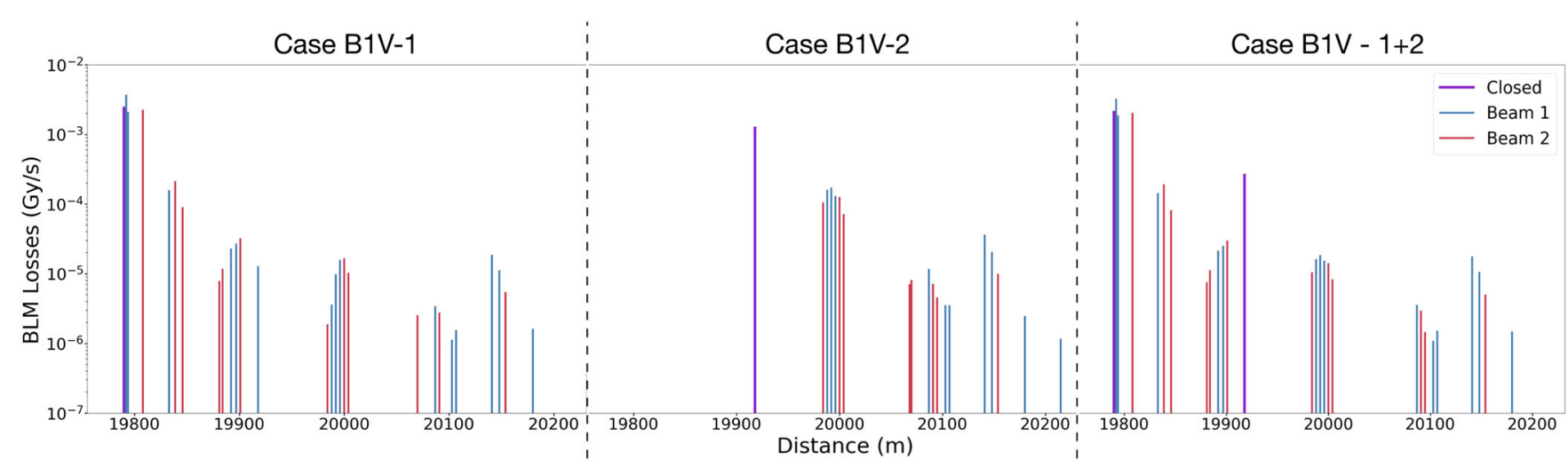

FIG. 5. The spatial distribution of the losses measured in IR7 using the three configurations of closed collimators in Fig. 4.
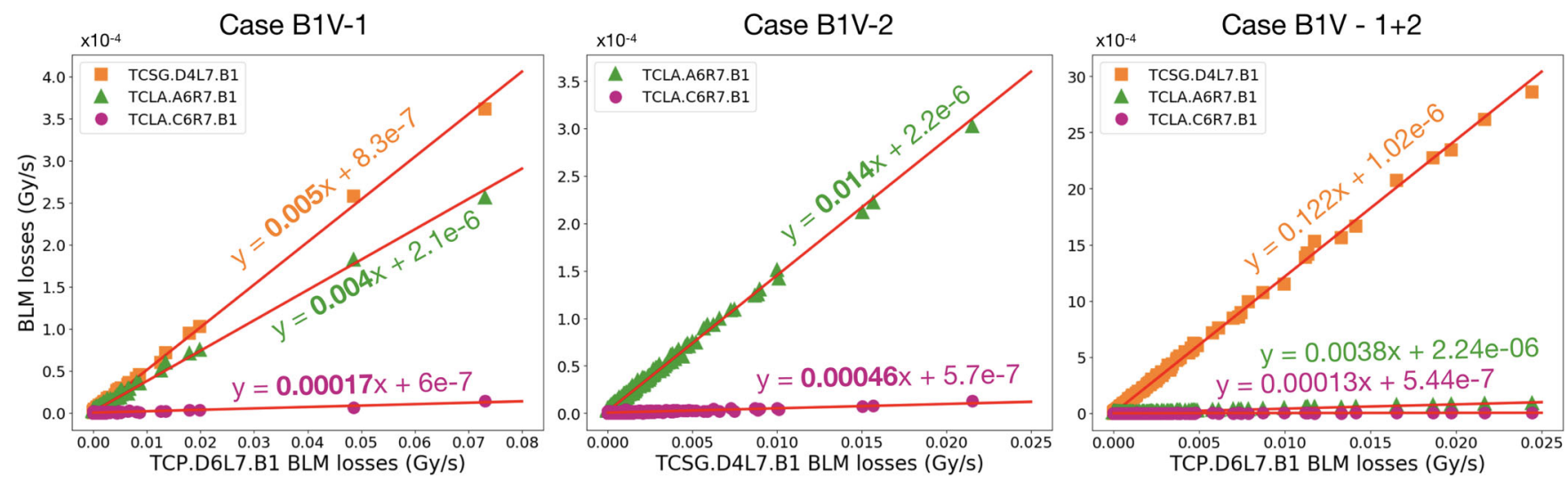

FIG. 6. The $100 \mathrm{~Hz}$ BLM signals recorded using the three configurations of closed collimators in Fig. 4, against the 100 Hz BLM signal of the first upstream collimator closed within the configuration. Linear fits were applied to each set of data points, and the gradients obtained using the individual case configurations are marked in bold.

of all the cross-talk factors is presented in Appendix A. The cross-talk factors confirm that the cross talk goes down the further downstream a collimator is located with respect to the primary loss location.

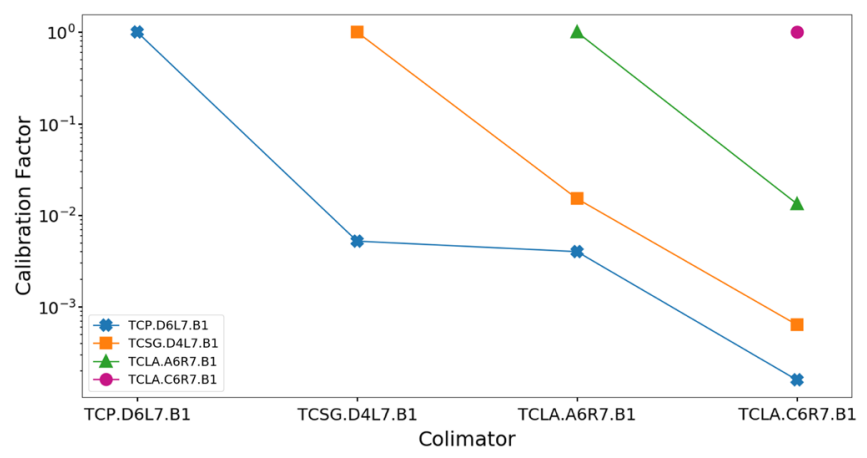

FIG. 7. The cross-talk factors quantifying the effect of each collimator on the downstream collimators, for the set of collimators used in the vertical plane in beam 1 (case B1V-1, 1, 0.005, 0.004, 0.00016; case B1V-2, 1, 0.015, 0.0006; case B1V-3, 1, 0.013 ; case B1V-4, 1).

\section{BLM COEFFICIENT VALIDATION}

The cross-talk factors determined for the respective collimator configurations are validated by comparing the losses predicted using the cross-talk factors to measurements for the 13 downstream collimators used. Figure 8 qualitatively compares the measured and predicted BLM signals for four of these configurations, one from each plane.

The overall results are displayed in Fig. 9, which shows all the measured signal points against their predictions. All points used are above $10^{-7} \mathrm{~Gy} / \mathrm{s}$, to exclude background noise. These results indicate a strong linear correlation between the two signals, especially as the signal increases. A linear fit was applied to the data with an $R^{2}$ of $98 \%$, showing that on average the predicted signal is $11 \%$ higher than the measured one. This could possibly be used to decrease the calculation error depicted in Fig. 10. The results are promising, as the error decreases systematically towards $10 \%$ for higher measured signals, whereas a larger error is expected with lower measured signals.

In the majority of the cases, the predicted signals are larger than the measured signals. This could be a result of 


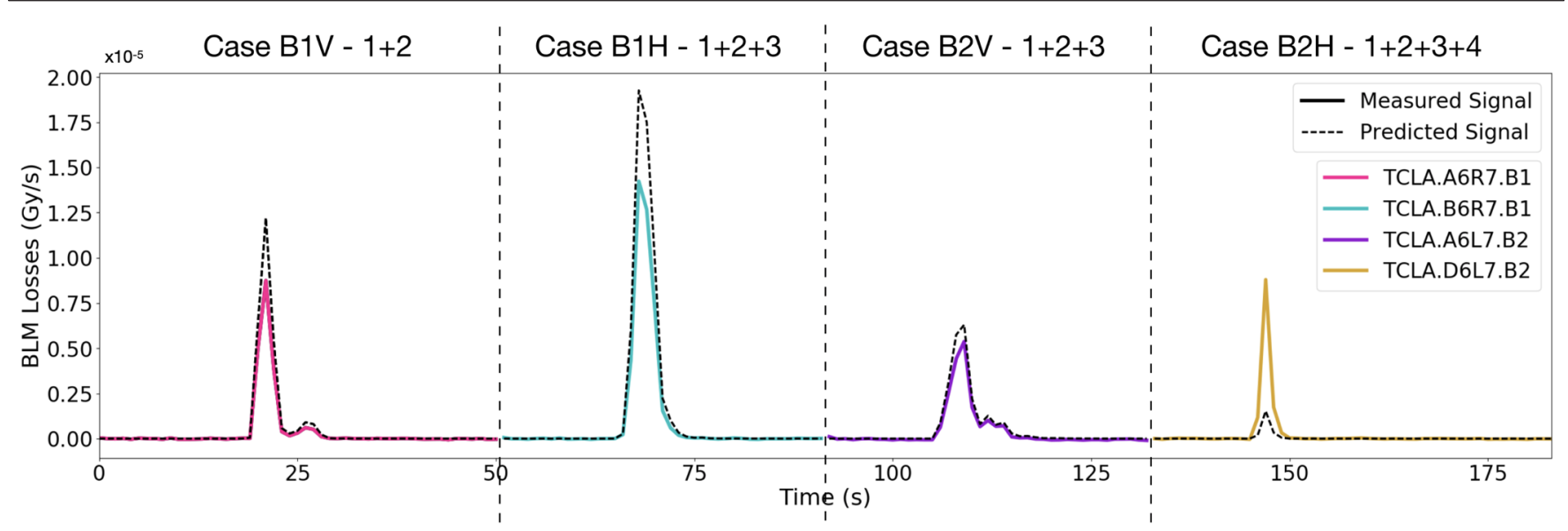

FIG. 8. A comparison of the measured and predicted signals of four configurations of closed collimators, one from each plane: case $\mathrm{B} 1 \mathrm{~V}-1+2$, case $\mathrm{B} 1 \mathrm{H}-1+2+3$, case $\mathrm{B} 2 \mathrm{~V}-1+2+3$, and case $\mathrm{B} 2 \mathrm{H}-1+2+3+4$.

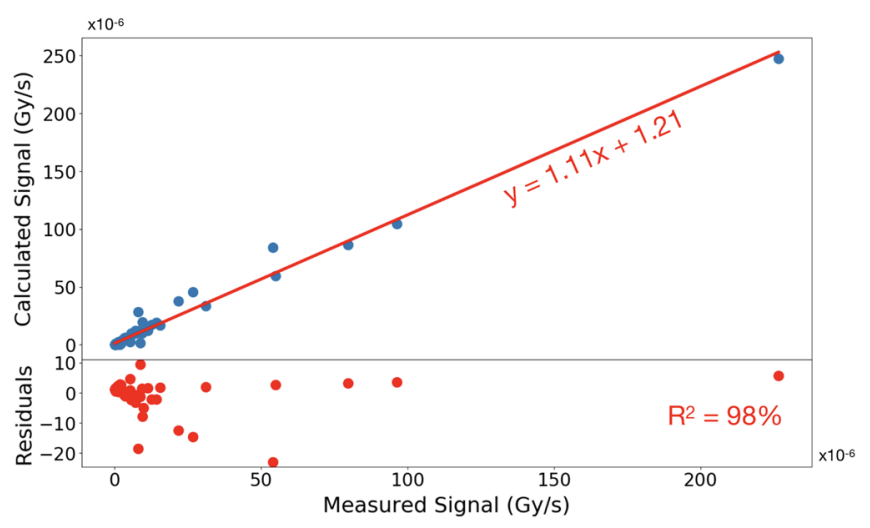

FIG. 9. Scatter plot of the measured signal points above $10^{-7} \mathrm{~Gy} / \mathrm{s}$, against the calculated signal points. A linear fit was applied to the data, and its residuals were also plotted.

upstream collimators shielding showers generated by impacts of particles on further upstream collimators, which are assumed to be zero in this paper. In addition, cases for which the predicted loss is smaller than the actual loss

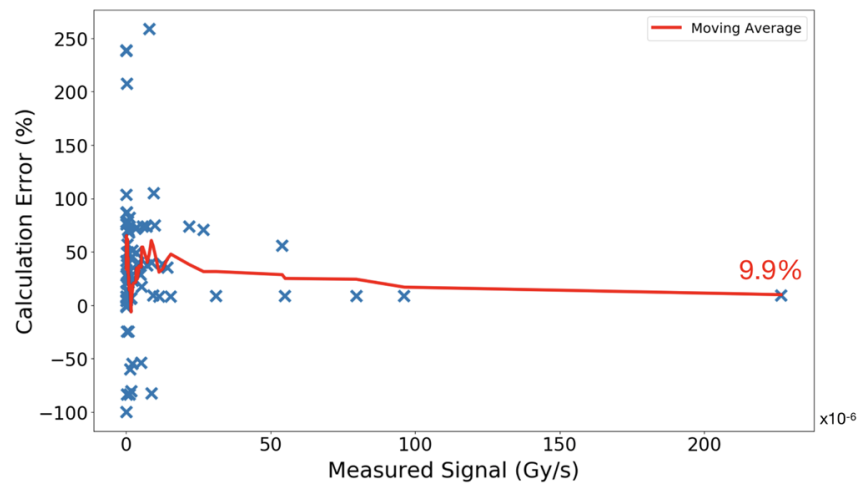

FIG. 10. Scatter plot of the measured signal points above $10^{-7} \mathrm{~Gy} / \mathrm{s}$, against the calculation error. The moving average was calculated showing the average error is mostly below $50 \%$ for low BLM signals and as small as $10 \%$ for high BLM signals. corresponded to signals below $10^{-5} \mathrm{~Gy} / \mathrm{s}$, which are more prone to errors. Therefore, overall, the results clearly indicate that it is indeed possible to estimate the cross talk that a collimator BLM will experience, within a reasonable margin.

\section{APPLICATION TO LOSS MAPS}

This work seeks to predict the losses at the first open collimator positioned downstream after a series of closed collimators; therefore, a case study for this analysis is an application to loss maps. During operation, two types of loss maps per beam are generated, horizontal and vertical loss maps, to examine the losses induced at the collimators by generating losses in the respective planes.

To apply this study to loss maps, the analysis can be used to predict the losses at the first skew collimator, as this is the plane in which no losses are generated, to mimic the requirement of having an "open" collimator. This can be calculated by applying the cross-talk factors in Appendix A to the losses at the horizontal and vertical collimators positioned upstream. Since no measurements were performed in the skew plane (recall Sec. II), no cross-talk factors were determined for that plane. According to the order the collimators are positioned around the beam (refer to Fig. 1), the collimators selected for this section are listed in Table IV.

The data used in this section were gathered from three loss map campaigns during LHC operation. The campaigns were selected such that the order of planes in which the losses were generated vary, to ensure no bias in the results.

TABLE IV. Collimator configurations used for skew collimator predictions in loss map case studies.

\begin{tabular}{lcc}
\hline \hline V case & H case & Skew \\
\hline B1V-1 +2 & B1H-1 +2 & TCSG.A4L7.B1 \\
B2V-1 $+2+3$ & B2H-1 +2 & TCSG.A4R7.B2 \\
\hline \hline
\end{tabular}




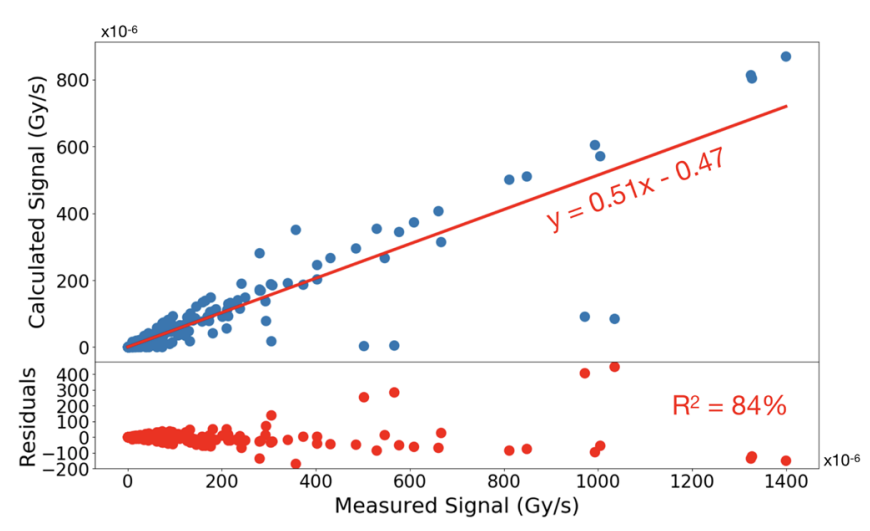

FIG. 11. Scatter plot of the loss map measured signal points above $10^{-7} \mathrm{~Gy} / \mathrm{s}$, against the calculated signal points, for the two skew collimators. A linear fit was applied to the data, and the fit residuals are presented in the lower plot.

Technical stop 2 in 2018.-After a technical stop, loss maps are used to validate the collimation hierarchy before resuming operation. In this case, loss maps were generated at injection and flattop, with the horizontal plane performed first.

Machine development (MD) time.-At the end of an MD at injection, time was dedicated to generate loss maps for this study. In this case, the vertical plane was specifically performed first.

Dedicated $M D$.- This was a dedicated test performed at flattop to generate losses by scraping the beam with the primary collimators. This scraping was performed to mimic the beam losses required for generating loss maps without using the standard hardware, to ensure that the order in which the losses are induced in the planes does not influence the results.

The individual configurations in Sec. II and the losses generated in the horizontal and vertical planes for the loss maps were used to predict the losses for the two skew collimators in both planes.

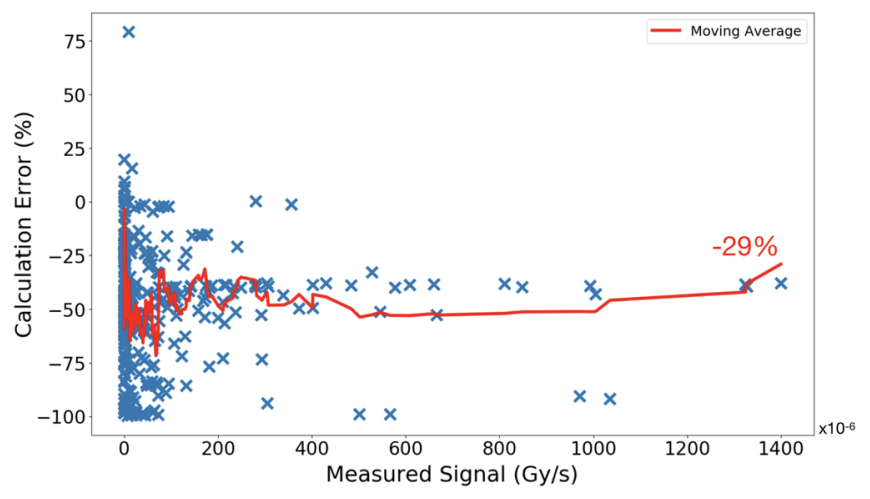

FIG. 12. Scatter plot of the loss map measured signal points above $10^{-7} \mathrm{~Gy} / \mathrm{s}$, against the calculation error, for the two skew collimators. The moving average was calculated, showing the average error is mostly around $-50 \%$ for low BLM signals and $-30 \%$ for high BLM signals.
The skew collimator results from the three loss map campaigns are displayed in Fig. 11, which plots the predicted signals against the measured signals, for the measured signal points above $10^{-7} \mathrm{~Gy} / \mathrm{s}$. A linear fit with an $R^{2}$ of $84 \%$ was applied to the data, and the calculation error is plotted in Fig. 12.

For the majority of cases, the calculated cross talk underestimates the measurements, with the average error starting off around $-50 \%$, and as the measured signal increases the error decreases to $-29 \%$. This is a result of the missing contribution from the skew collimators when calculating the cross talk, as explained previously.

\section{CALCULATION OF PROTON IMPACTS}

Having successfully quantified the level of cross-talk observed by BLMs in Sec. V, this section seeks to calculate the equivalent impact rate for any collimator $j$, in protons per second $\left(P_{j}\right)$. Following the same approach as in Sec. III, the signals of all closed upstream collimators must be taken into consideration using Eq. (1). This is transformed into Eq. (6) to calculate the effect of a single collimator on its BLM:

$$
\begin{aligned}
& S_{n}=\sum_{i=1}^{n-1} a_{n i} \cdot S_{i}^{\prime}+a_{n n} S_{n}^{\prime}, \\
& a_{n n}=1, \quad S_{n}^{\prime}=S_{n}-\sum_{i=1}^{n-1} a_{n i} \cdot S_{i}^{\prime} .
\end{aligned}
$$

TABLE V. Cross-talk factors (protons per Gy), calculated from the change in beam intensity using the individual cases.

\begin{tabular}{lcc}
\hline \hline Plane & Collimator & Factor \\
\hline B1V & TCP.D6L7.B1 & $1.17 \times 10^{12}$ \\
& TCSG.D4L7.B1 & $9.36 \times 10^{11}$ \\
& TCLA.A6R7.B1 & $5.72 \times 10^{11}$ \\
TCLA.C6R7.B1 & $9.43 \times 10^{11}$ \\
B1H & TCP.C6L7.B1 & $7.95 \times 10^{11}$ \\
& TCSG.B4L7.B1 & $8.66 \times 10^{11}$ \\
& TCSG.6R7.B1 & $6.48 \times 10^{11}$ \\
& TCLA.B6R7.B1 & $4.39 \times 10^{11}$ \\
& TCLA.D6R7.B1 & $6.72 \times 10^{11}$ \\
B2V & TCLA.A7R7.B1 & $6.59 \times 10^{11}$ \\
& TCP.D6R7.B2 & $9.54 \times 10^{11}$ \\
& TCSG.D4R7.B2 & $7.32 \times 10^{11}$ \\
& TCSPM.D4R7.B2 & $7.04 \times 10^{11}$ \\
& TCLA.A6L7.B2 & $5.58 \times 10^{11}$ \\
B2H & TCLA.C6L7.B2 & $6.61 \times 10^{11}$ \\
& TCP.C6R7.B2 & $9.66 \times 10^{11}$ \\
& TCSG.B4R7.B2 & $8.56 \times 10^{11}$ \\
& TCSG.6L7.B2 & $7.56 \times 10^{11}$ \\
& TCLA.B6L7.B2 & $5.12 \times 10^{11}$ \\
& TCLA.D6L7.B2 & $1.34 \times 10^{13}$ \\
& TCLA.A7L7.B2 & $2.85 \times 10^{11}$ \\
\hline \hline
\end{tabular}




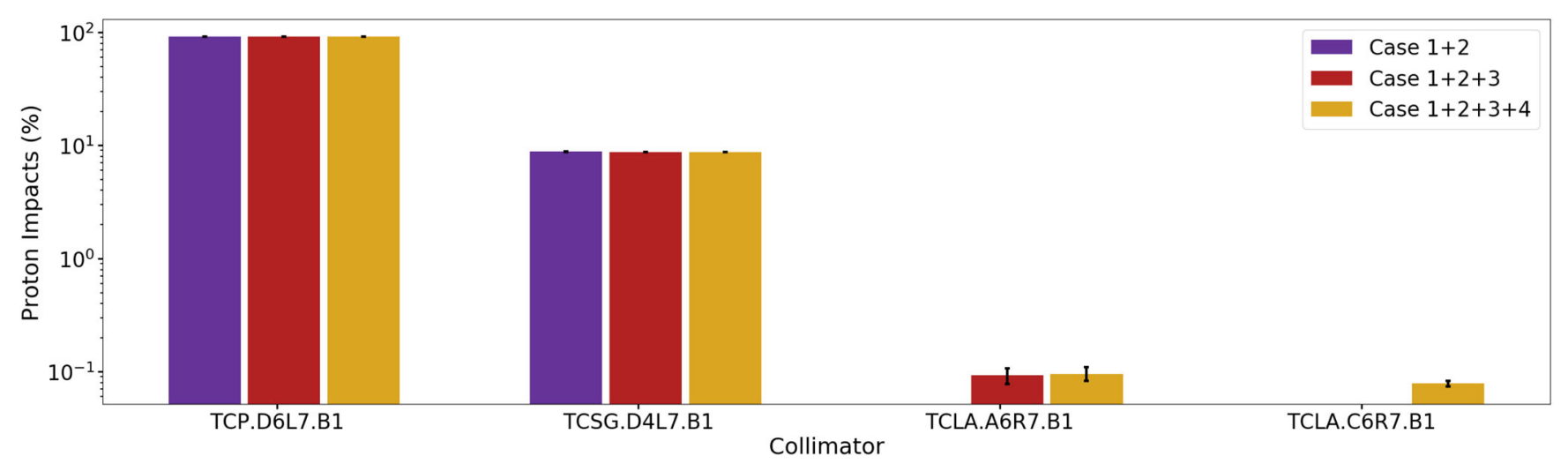

FIG. 13. The percentage distribution of the predicted proton impacts for the three configurations of closed collimators in the vertical plane in beam 1: case B1V-1 +2, case B1V-1 + 2+3, and case B1V-1 + $2+3+4$.

This is then extended into

$$
P_{n}=b_{n} . S_{n}^{\prime}, \quad b_{n}=\frac{\Delta I}{S_{n}},
$$

where $b^{n}$ is the cross-talk factor in protons per Gy of collimator $n$, calculated from the change in beam intensity $(\Delta I)$ against the BLM signal of collimator $n$, when it was the only one closed (case $n$ ). The cross-talk factors of all collimators are collected in Table V.

The protons impacting each collimator $i$ can be compared by representing them as a percentage of the total impacts per combined case (case $1+\cdots+n$ ), using

$$
P_{i}^{\prime}=\frac{P_{i}}{\sum_{j=1}^{n} P_{j}} \times 100, \quad i \leq n
$$

The proton impacts calculated for the three combined cases in the vertical plane in beam 1 are displayed in Fig. 13, indicating that $91 \%$ of all protons lost are assigned to the primary collimator, $9 \%$ are assigned to the secondary collimator, and the rest are below $0.001 \%$.

\section{SIMULATED PROTON IMPACTS}

To validate the calculated proton impacts, the results are compared to simulated impacts. The simulations were run using SIXTRACK [7], which is the standard simulation tool used at CERN for tracking particles in the accelerator. The simulations were set up using the methods described in Ref. [8], as this type of simulation has shown a good agreement with measurements in previous studies [8-10]. The simulations were set to use the collimator positions (refer to Table II) and beam optics that were used during the beam tests.

The results for the vertical plane in beam 1 are displayed in Fig. 14, which overlays the simulated impacts on the measured impacts (refer to Appendix B for a complete table of all the calculated impacts). From the simulations, 94\% of all protons lost are assigned to the primary collimator, $6 \%$ are assigned to the secondary collimator, and the rest are below $0.001 \%$, indicating a $\pm 3 \%$ difference from the measured impacts. Overall, the two sets of impact results are similar; however, one can notice that the overestimation in the measured impacts increases the further downstream a collimator is. This is another effect of upstream collimators shielding showers before they reach the downstream collimators, which are assumed to be zero in this paper.

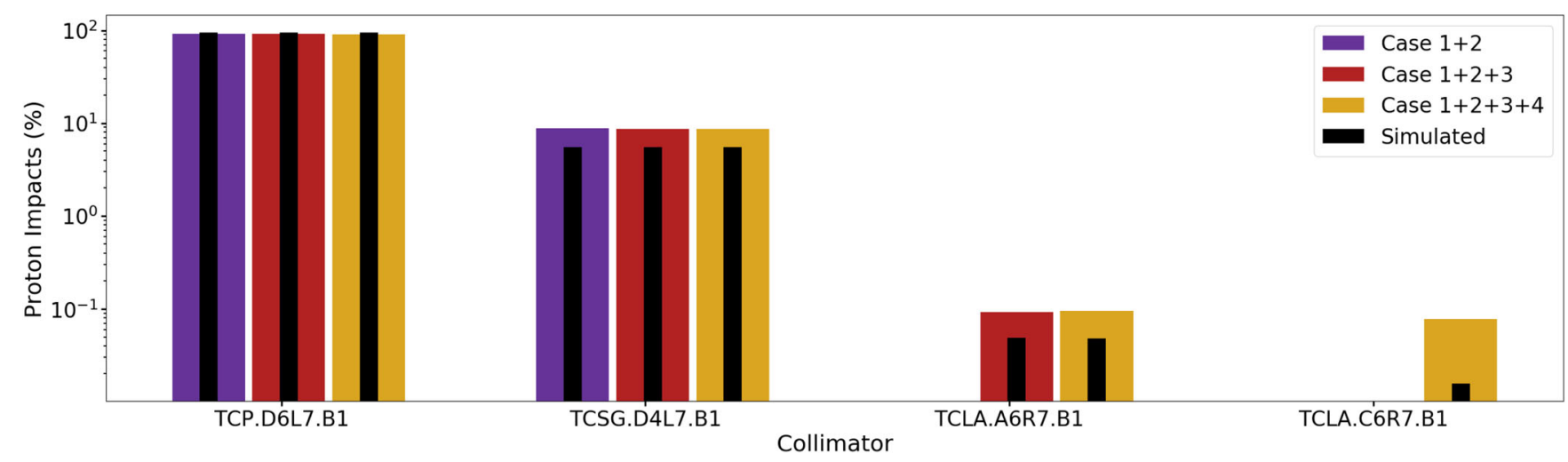

FIG. 14. A comparison of the percentage distribution of the predicted and simulated proton impacts for the three configurations in the vertical plane in beam 1 : case $\mathrm{B} 1 \mathrm{~V}-1+2$, case $\mathrm{B} 1 \mathrm{~V}-1+2+3$, and case $\mathrm{B} 1 \mathrm{~V}-1+2+3+4$. 


\section{CONCLUSION}

Collimators are often aligned sequentially to avoid any cross talk between the beam loss monitors, as this may lead to errors in determining the beam center and beam size at the collimator. This paper seeks to quantify the levels of cross-talk observed by beam loss monitors to determine the actual beam loss signals generated by their corresponding collimators.

A number of beam tests were performed for various combinations of closed IR7 collimators, in the horizontal and vertical planes. These were used to predict the amount of cross-talk observed at the first open downstream collimator in each case. The cross talk was successfully predicted with an error less than $10 \%$ of the measured BLM signal. In most cases, the cross talk was overestimated as the showers shielded by upstream collimators were assumed to be zero in this paper.

These results were then applied to loss maps, to predict the losses at the first skew collimator positioned downstream, when losses are generated in the horizontal and vertical planes. The results indicate that the amount of cross talk was underestimated for the majority of the cases. This is due to the fact that the contributions of the skew collimators during the loss maps were not included, as no information was gathered for skew collimators during the beam tests for this study.

Finally, the number of protons that impacted each collimator were then calculated for each of the combined cases of closed collimators. The impacts at the primary collimators are conclusive, as they are equal to the change in the beam intensity. On the other hand, the losses at downstream collimators overestimate the amount of proton impacts, due to the showers shielded by upstream collimators assumed to be zero.

This study provides a foundation for cross-talk analysis across BLMs in LHC collimators which can be further expanded by taking measurements using more collimators, especially skew collimators. This can also be extended by repeating the measurements at flattop and analyzing any energy-dependent effects.

\section{ACKNOWLEDGMENTS}

The authors acknowledge the support of D. Mirarchi, J. Molson, and A. Mereghetti from the collimation team, for their help in setting up the simulations and the many discussions on analyzing the simulated data.

\section{APPENDIX A: CROSS-TALK FACTORS}

This Appendix gathers, in Table VI, cross-talk factors measured from beam tests, which quantify the effect of a closed collimator on all downstream collimators.

TABLE VI. BLM cross-talk factors calculated from the individual cases during LHC beam tests.

\begin{tabular}{|c|c|c|c|c|c|c|}
\hline \multirow[t]{2}{*}{ Case ID } & \multicolumn{6}{|c|}{ Affected collimators } \\
\hline & TCP.D6L7.B1 & TCSG.D4L7.B1 & TCLA.A6R7.B1 & TCLA.C6R7.B1 & & \\
\hline B1V-1 & 1.0 & 0.00523 & 0.00402 & 0.00016 & & \\
\hline B1V-2 & $\cdots$ & 1.0 & 0.01522 & 0.00064 & & \\
\hline B1V-3 & $\cdots$ & $\ldots$ & 1.0 & 0.01340 & & \\
\hline B1V-4 & $\cdots$ & $\cdots$ & $\cdots$ & 1.0 & & \\
\hline & TCP.C6L7.B1 & TCSG.B4L7.B1 & TCSG.6R7.B1 & TCLA.B6R7.B1 & TCLA.D6R7.B1 & TCLA.A7R7.B1 \\
\hline B1H-1 & 1.0 & 0.00093 & 0.014584 & 0.00094 & 0.00039 & 0.00018 \\
\hline B1H-2 & $\cdots$ & 1.0 & 0.013217 & 0.00131 & 0.00284 & 0.00022 \\
\hline B1H-3 & $\cdots$ & $\cdots$ & 1.0 & 0.05542 & 0.02536 & 0.00730 \\
\hline B1H-4 & $\cdots$ & $\cdots$ & $\cdots$ & 1.0 & 0.02127 & 0.00831 \\
\hline B1H-5 & $\cdots$ & $\cdots$ & $\cdots$ & $\cdots$ & 1.0 & 0.01688 \\
\hline В1H-6 & $\cdots$ & $\cdots$ & $\cdots$ & $\cdots$ & $\cdots$ & 1.0 \\
\hline & TCP.D6R7.B2 & TCSG.D4R7.B2 & TCSPM.D4R7.B2 & TCLA.A6L7.B2 & TCLA.C6L7.B2 & \\
\hline B2V-1 & 1.0 & 0.0052 & 0.00304 & 0.00257 & 0.00016 & \\
\hline B2V-2 & $\ldots$ & 1.0 & 0.61890 & 0.00761 & 0.00020 & \\
\hline $\mathrm{B} 2 \mathrm{~V}-3$ & $\cdots$ & $\cdots$ & 1.0 & 0.00979 & 0.00030 & \\
\hline B2V-4 & $\cdots$ & $\cdots$ & $\cdots$ & 1.0 & 0.01237 & \\
\hline B2V-5 & $\cdots$ & $\cdots$ & $\cdots$ & $\cdots$ & 1.0 & \\
\hline & TCP.C6R7.B2 & TCSG.B4R7.B2 & TCSG.6L7.B2 & TCLA.B6L7.B2 & TCLA.D6L7.B2 & TCLA.A7L7.B2 \\
\hline B2H-1 & 1.0 & 0.00105 & 0.01836 & 0.00122 & 0.00039 & 0.00018 \\
\hline B2H-2 & $\cdots$ & 1.0 & 0.01432 & 0.00165 & 0.00217 & 0.00033 \\
\hline B2H-3 & $\cdots$ & $\cdots$ & 1.0 & 0.08164 & 0.03403 & 0.01557 \\
\hline В2H-4 & $\cdots$ & $\cdots$ & $\cdots$ & 1.0 & 0.02399 & 0.01535 \\
\hline B2H-5 & $\cdots$ & $\cdots$ & $\cdots$ & $\cdots$ & 1.0 & 0.02145 \\
\hline В2H-6 & $\cdots$ & $\cdots$ & $\cdots$ & $\cdots$ & $\cdots$ & 1.0 \\
\hline
\end{tabular}




\section{APPENDIX B: MEASURED AND SIMULATED IMPACTS}

This Appendix gathers, in Table VII, the percentage distribution of proton impacts that were measured from beam tests, compared to SiXTRACK simulation results. Due to the shielding of upstream collimators assumed to be zero, the proton impacts at all downstream collimators are overestimated, thus underestimating the percentage of impacts at the primary collimators.

TABLE VII. Percentage of proton impacts measured and simulated from the combined cases of closed collimators.

\begin{tabular}{|c|c|c|c|c|c|c|}
\hline \multirow[t]{2}{*}{ Case ID } & \multicolumn{6}{|c|}{ Measured collimator impacts } \\
\hline & TCP.D6L7 & TCSG.D4L7 & TCLA.A6R7 & TCLA.C6R7 & & \\
\hline $\mathrm{B} 1 \mathrm{~V}-1+2$ & 0.91244 & 0.08756 & $\ldots$ & $\ldots$ & & \\
\hline $\mathrm{B} 1 \mathrm{~V}-1+2+3$ & 0.91201 & 0.08706 & 0.00092 & $\ldots$ & & \\
\hline \multirow[t]{2}{*}{$\mathrm{B} 1 \mathrm{~V}-1+\cdots+4$} & 0.91121 & 0.08704 & 0.00096 & 0.00078 & & \\
\hline & TCP.C6L7 & TCSG.B4L7 & TCSG.6R7 & TCLA.B6R7 & TCLA.D6R7 & TCLA.A7R7 \\
\hline $\mathrm{B} 1 \mathrm{H}-1+2$ & 0.78973 & 0.21027 & $\ldots$ & $\ldots$ & $\ldots$ & $\ldots$ \\
\hline B1H-1 + $2+3$ & 0.74165 & 0.17754 & 0.08081 & $\ldots$ & $\cdots$ & $\cdots$ \\
\hline $\mathrm{B} 1 \mathrm{H}-1+\cdots+4$ & 0.74997 & 0.17338 & 0.07406 & 0.00258 & $\ldots$ & $\cdots$ \\
\hline $\mathrm{B} 1 \mathrm{H}-1+\cdots+5$ & 0.74876 & 0.17105 & 0.07724 & 0.00261 & 0.00033 & $\cdots$ \\
\hline \multirow[t]{2}{*}{$\mathrm{B} 1 \mathrm{H}-1+\cdots+6$} & 0.75561 & 0.16990 & 0.07141 & 0.00224 & 0.00042 & 0.00042 \\
\hline & TCP.D6R7 & TCSG.D4R7 & TCSPM.D4R7 & TCLA.A6L7 & TCLA.C6L7 & \\
\hline $\mathrm{B} 2 \mathrm{~V}-1+2$ & 0.88429 & 0.11571 & $\ldots$ & $\ldots$ & $\ldots$ & \\
\hline $\mathrm{B} 2 \mathrm{~V}-1+2+3$ & 0.82250 & 0.11091 & 0.06659 & $\ldots$ & $\cdots$ & \\
\hline $\mathrm{B} 2 \mathrm{~V}-1+\cdots+4$ & 0.82752 & 0.10763 & 0.06468 & 0.00017 & $\ldots$ & \\
\hline \multirow{2}{*}{$\mathrm{B} 2 \mathrm{~V}-1+\cdots+5$} & 0.82376 & 0.10759 & 0.06489 & 0.00365 & 0.00011 & \\
\hline & TCP.C6R7 & TCSG.B4R7 & TCSG.6L7 & TCLA.B6L7 & TCLA.D6L7 & TCLA.A7L7 \\
\hline $\mathrm{B} 2 \mathrm{H}-1+2$ & 0.75050 & 0.24950 & $\ldots$ & $\ldots$ & $\ldots$ & $\ldots$ \\
\hline $\mathrm{B} 2 \mathrm{H}-1+2+3$ & 0.70668 & 0.15890 & 0.13441 & $\ldots$ & $\ldots$ & $\ldots$ \\
\hline $\mathrm{B} 2 \mathrm{H}-1+\cdots+4$ & 0.70038 & 0.16475 & 0.13472 & 0.00015 & $\cdots$ & $\cdots$ \\
\hline $\mathrm{B} 2 \mathrm{H}-1+\cdots+5$ & 0.69905 & 0.16281 & 0.13738 & 0.00076 & 0 & $\ldots$ \\
\hline $\mathrm{B} 2 \mathrm{H}-1+\cdots+6$ & 0.69675 & 0.16309 & 0.13939 & 0.00058 & 0 & 0.00019 \\
\hline \multirow[t]{2}{*}{ Case ID } & \multicolumn{6}{|c|}{ Simulated collimator impacts } \\
\hline & TCP.D6L7 & TCSG.D4L7 & TCLA.A6R7 & TCLA.C6R7 & & \\
\hline $\mathrm{B} 1 \mathrm{~V}-1+2$ & 0.94446 & 0.05552 & $\ldots$ & $\ldots$ & & \\
\hline $\mathrm{B} 1 \mathrm{~V}-1+2+3$ & 0.94420 & 0.05530 & 0.00049 & $\ldots$ & & \\
\hline \multirow[t]{2}{*}{$\mathrm{B} 1 \mathrm{~V}-1+\cdots+4$} & 0.94395 & 0.05541 & 0.00048 & 0.00016 & & \\
\hline & TCP.C6L7 & TCSG.B4L7 & TCSG.6R7 & TCLA.B6R7 & TCLA.D6R7 & TCLA.A7R7 \\
\hline $\mathrm{B} 1 \mathrm{H}-1+2$ & 0.88632 & 0.10943 & $\cdots$ & $\ldots$ & $\cdots$ & $\ldots$ \\
\hline $\mathrm{B} 1 \mathrm{H}-1+2+3$ & 0.85836 & 0.07169 & 0.06919 & $\ldots$ & $\cdots$ & $\cdots$ \\
\hline $\mathrm{B} 1 \mathrm{H}-1+\cdots+4$ & 0.85697 & 0.07164 & 0.06909 & 0.00216 & $\ldots$ & $\cdots$ \\
\hline $\mathrm{B} 1 \mathrm{H}-1+\cdots+5$ & 0.85627 & 0.07161 & 0.06894 & 0.00217 & 0.00102 & $\ldots$ \\
\hline \multirow{2}{*}{$\mathrm{B} 1 \mathrm{H}-1+\cdots+6$} & 0.85592 & 0.07154 & 0.06897 & 0.00214 & 0.00101 & 0.00042 \\
\hline & TCP.D6R7 & TCSG.D4R7 & TCSPM.D4R7 & TCLA.A6L7 & TCLA.C6L7 & \\
\hline B2V-1 + 2 & 0.93951 & 0.06049 & $\ldots$ & $\ldots$ & $\ldots$ & \\
\hline $\mathrm{B} 2 \mathrm{~V}-1+2+3$ & 0.93477 & 0.06004 & 0.00519 & $\ldots$ & $\cdots$ & \\
\hline $\mathrm{B} 2 \mathrm{~V}-1+\cdots+4$ & 0.93450 & 0.06001 & 0.00518 & 0.00031 & $\ldots$ & \\
\hline \multirow[t]{2}{*}{$\mathrm{B} 2 \mathrm{~V}-1+\cdots+5$} & 0.93447 & 0.06001 & 0.00519 & 0.00032 & 0.000004 & \\
\hline & TCP.C6R7 & TCSG.B4R7 & TCSG.6L7 & TCLA.B6L7 & TCLA.D6L7 & TCLA.A7L7 \\
\hline $\mathrm{B} 2 \mathrm{H}-1+2$ & 0.89701 & 0.09678 & $\ldots$ & $\ldots$ & $\ldots$ & $\ldots$ \\
\hline B2H-1 + $2+3$ & 0.87597 & 0.06427 & 0.05878 & $\ldots$ & $\cdots$ & $\cdots$ \\
\hline B2H-1 $+\cdots+4$ & 0.87511 & 0.06415 & 0.05864 & 0.001838 & $\ldots$ & $\cdots$ \\
\hline $\mathrm{B} 2 \mathrm{H}-1+\cdots+5$ & 0.87464 & 0.06415 & 0.05877 & 0.001829 & 0.00079 & $\ldots$ \\
\hline $\mathrm{B} 2 \mathrm{H}-1+\cdots+6$ & 0.87426 & 0.06418 & 0.05858 & 0.001832 & 0.00079 & 0.00035 \\
\hline
\end{tabular}


[1] Lyndon Evans, The Large Hadron Collider, New J. Phys. 9, 335 (2007).

[2] L. R. Evans, Beam physics at LHC, in Proceedings of the 2003 Particle Accelerator Conference, Portland, OR (IEEE, New York, 2003), Vol. 1, pp. 19-23.

[3] R. W. Assmann et al., Requirements for the LHC collimation system, Technical Report No. CERN-LHC-ProjectReport-599, CERN, 2002.

[4] E. B. Holzer et al., Beam loss monitoring system for the LHC, in Proceedings of the IEEE Nuclear Science Symposium Conference Record, 2005 (IEEE, New York, 2005), Vol. 2, pp. 1052-1056.

[5] G. Azzopardi, B. M. Salvachua Ferrando, and G. Valentino, MD1653-Part 1: Characterisation of BLM response at collimators, Technical Report No. CERNACC-NOTE-2018-0070, CERN, 2018.

[6] W. Hofle et al., Controlled transverse blow-up of highenergy proton beams for aperture measurements and loss maps, in Proceedings of the 3rd International Particle
Accelerator Conference, New Orleans, LA, 2012 (IEEE, Piscataway, NJ, 2012), Vol. 12, pp. 4059-4061.

[7] F. Schmidt, SiXtrack: Version 4.2.16 single particle tracking code treating transverse motion with synchrotron oscillations in a symplectic manner-User's reference manual, update, 2012.

[8] R. Bruce et al., Simulations and measurements of beam loss patterns at the CERN Large Hadron Collider, Phys. Rev. ST Accel. Beams 17, 081004 (2014).

[9] R. Bruce, C. Bracco, R. De Maria, M. Giovannozzi, A. Mereghetti, D. Mirarchi, S. Redaelli, E. Quaranta, and B. Salvachua, Reaching record-low $\beta^{*}$ at the CERN Large Hadron Collider using a novel scheme of collimator settings and optics, Nucl. Instrum. Methods Phys. Res., Sect. A 848, 19 (2017).

[10] R. Bruce et al., Collimation-induced experimental background studies at the CERN Large Hadron Collider, Phys. Rev. Accel. Beams 22, 021004 (2019). 